\title{
Antenna Performance Improvement Techniques for Energy Harvesting: A Review Study
}

\author{
Raed Abdulkareem Abdulhasan*, Abdulrashid O. Mumin, Yasir A. Jawhar, Mustafa S. Ahmed, Rozlan Alias, \\ Khairun Nidzam Ramli, Mariyam Jamilah Homam and Lukman Hanif Muhammad Audah \\ Faculty of Electrical and Electronics Engineering, Universiti Tun Hussein Onn Malaysia, \\ 86400, Parit Raja, Batu Pahat, Johor, Malaysia
}

\begin{abstract}
The energy harvesting is defined as using energy that is available within the environment to increase the efficiency of any application. Moreover, this method is recognized as a useful way to break down the limitation of battery power for wireless devices. In this paper, several antenna designs of energy harvesting are introduced. The improved results are summarized as a $2 \times 2$ patch array antenna realizes improved efficiency by 3.9 times higher than the single patch antenna. The antenna has enhanced the bandwidth of $22.5 \mathrm{MHz}$ after load two slots on the patch. The solar cell antenna is allowing harvesting energy during daylight. A couple of E-patches antennas have increased the bandwidth of $33 \%$ and the directivity up to $20 \mathrm{dBi}$. The received power can be improved by 1.2-1.4 times when using the dual port on pixel antenna. Complementary split ring resonator and substrate integrated waveguide are utilized cavity-backed feeding on a fractal patch antenna to enhance the bandwidth around $5.1 \%$. Moreover, adding a rectifier circuit to an antenna converts the reserved RF-signal to DC power, and then duplicated the input voltage up to sum the total number of rectifier circuit stages. Therefore, the advantages and disadvantages of each antenna depend on the technique which used in design.
\end{abstract}

Keywords-energy harvesting; slotted patch; circularly polarization; solar substrate; rectenna

\section{INTRODUCTION}

Nowadays, many researchers focus on saving power and energy for modern applications [1]. In communications systems, the energy conservation grows to be a hot study. Moreover, the designers are looking for reducing the operation power in wireless applications. For example, medical testing devices and wireless sensor network (WSN) have a limited battery capacity. Complicity process encountered to replace the battery due to the location of the sensor inside the patient's body. Therefore, many researchers have developed the energy harvesting $(\mathrm{EH})$ techniques to solve this matter in communications devices. As a result, the sensors can keep working even after wasting the power from the battery. In 2016, Shaikh demonstrated several types of harvested energy, which are continuous or limited, natural or dependent, and environment or external sources [2]. Moreover, it is imperative to choose the type of the harvested energy, and any techniques are possible to use. Especially in wireless receiver devices, it is essential to improve the system efficiency, the performance effectiveness, and practical life. Enough energy can store it into a capacitor. It may lead to reducing the cost of the rechargeable

The authors would like to thank University Tun Hussein Onn Malaysia (UTHM) for sponsoring this work under this job under for Postgraduate Research Assistance Grant (GPPS) U464. battery [3, 4]. Replacing the device's battery is a challenging task to do.

The sensor nodes are used to transmit the data information. The researchers may set sensors on a difficult terrain, such as volcanoes and mountains. Therefore, it is costly to recharge the power. In this case, it is required to keep the sensors working for a longer time by harvesting the energy [5]. In that situation, the energy harvesting will increase the default lifetime for different wireless applications. Thus, this paper reviews different techniques of energy harvesting on antenna design. Moreover, these techniques have a significant influence to convert the energy and increase the efficiency of wireless devices.

Regarding our study, we give a summary of multiple ideas to improve antennas and harvesting energy. The methods of this paper are organized as follows: Phase controlling, slots on the antenna, enhance the antenna gain by using a reflector, solar cell, coupled E-patch antenna, dual-port feeding, substrate integrated waveguide, and rectenna.

\section{MODELING OF ENERGY HARVESTING}

All the works on antenna energy harvesting were carried out to give smart applications with high efficient transmitted power, and low-cost designing apply to antenna devices. The main advantages for $\mathrm{EH}$ could be summarized as the following: saving the authority to use it in case the devices have a little power and increase the battery end lifetime. To focus on this, we reviewed different techniques and analyzed of the characteristic of them.

\section{A. Phase control for microstrip patch array antenna}

Yang published an article [6]. Moreover, it was described the energy harvesting by controlling the phase shift to developing a $2 \times 2$ patch array antenna at $0.915 \mathrm{GHz}$. In the results, an increment RF power and constricts the energy for an array antenna. The result shows the efficiency improved by 3.9 times higher than one patch antenna at the same range and the supplied power. Furthermore, controlling the phase shift could enhance the effectiveness and variety the beam forming up to $\pm 15^{\circ}$.

In this paper, the authors determined the beam forming for a transmitter. A quarter of the power, which was collected from the receiver the single patch power, was less than the array antenna to provide the RF power. The four patches ordered a probe feed for the prototype array antenna. Moreover, only 
single patch antenna was chosen to draw the radiation pattern. The input factor of the array antenna should be considered to adjust the distance between the patches. Approximately, $\lambda / 2$ it is the best distance between the array patches during the simulation. The array antenna has a simulated gain around (3 $\mathrm{dB}$ ), which is higher than the gain of using one patch antenna. Tuning the phase shift for providing a signal from the antenna could realize the beam forming. Fig. 1 illustrates a systematically $90^{\circ}$ phase shift.

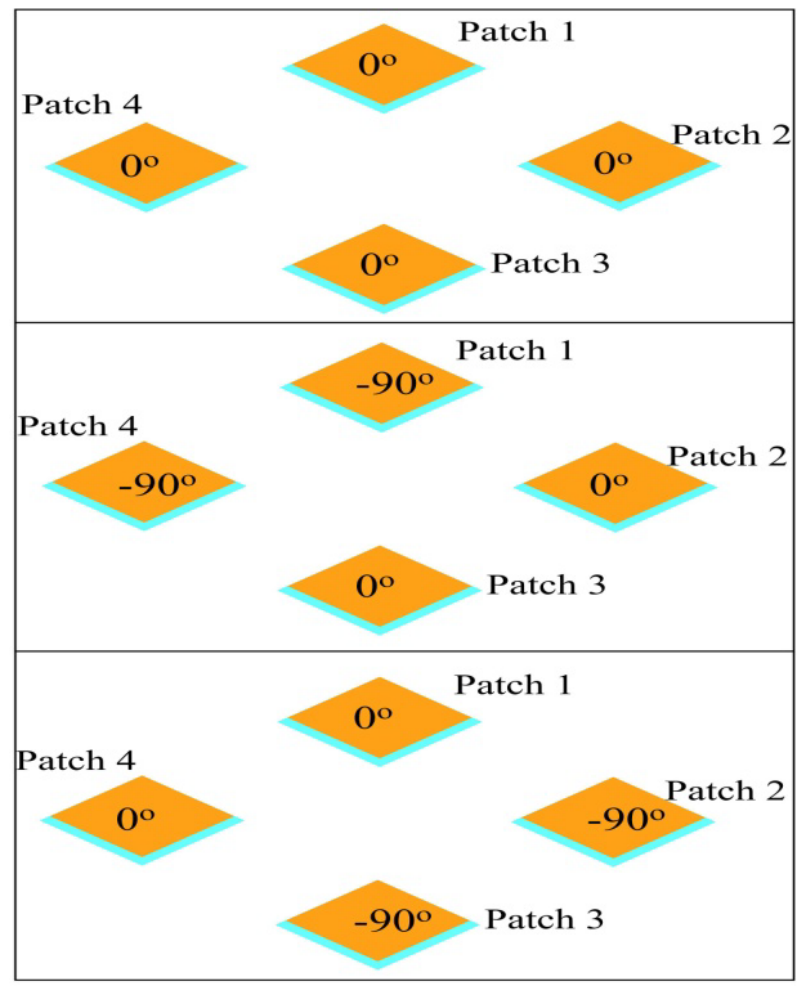

Fig. 1. $90^{\circ}$ phase shift applied [6]

In this research, the authors developed a phase shift factor. The best achievement target is to satisfy $360^{\circ}$ beam stern. LC should have $\lambda / 4$ adjustable load impedance in series to resonant the phase. The benefit of energy harvesting here is summarized by controlling the beam forming of emitted power. In short, $\pm 15^{\circ}$ the angle can be stern the central axis of the transmitted beam by varying $90^{\circ}$ phase shift between the antennas.

\section{B. Slots enhancement of antenna bandwidth}

On the other side, Moura Tiago demonstrated slots, which loaded on parasitic patch antenna for EH application [7]. This antenna had two slots at $754 \mathrm{MHz}$ to enhance the gain and bandwidth. It presented a greater limitation of a conventional patch antenna. The simulation result illustrates the bandwidth improved by $17 \mathrm{MHz}$. However, the experimental bandwidth for the demonstrated antenna was $22.5 \mathrm{MHz}$. Fig. 2 presents a couple of rectangular slots. DTT channel was considered by improving the bandwidth of the proposed antenna. From the basic theoretical information, the bandwidth of an antenna could be improved significantly by applying the slots on the patch. The researcher variation sets correctly from the designer. These two resonant bandwidths become wider and enhanced by using this configuration. The two slots improved the bandwidth by $5.1 \mathrm{MHz}$. This method gives an efficient improvement compared with the reference antenna bandwidth. There is no enhancement on the antenna gain by this technique. A high-gain antenna will improve the total energy harvesting of all systems and increase the efficiency significantly. So that, many researchers developed the parasitic stacked technique to enhance the bandwidth and gain together. The most significant effect parameters of impedance matching are the ground plane length, the feed line width, and width of the substrate [8,9]. Fig.3 illustrates the parasitic stacked patch antenna. It previously shows the slot patch feeding in Fig. 2. Indeed, the wavelength of the antenna is related to the dielectric of the substrate, and the gap between the coaxial feeding patch with the patristic patch, which has a height $\mathrm{H}$. The optimizing techniques and calculation have been considered to calculate the air gap $\mathrm{H}=25 \mathrm{~mm}$. The approximation height $H$ between $0.05 \lambda_{0}$ and $0.15 \lambda_{0}$ were found on academic references.

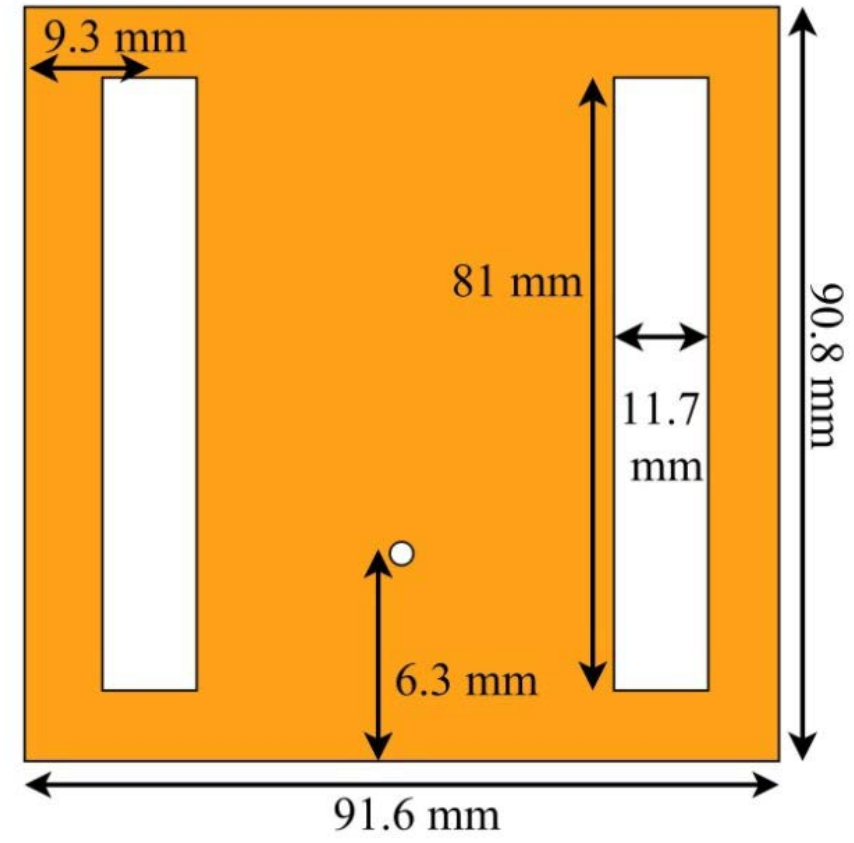

Fig. 2. Slot patch antenna configuration [7]

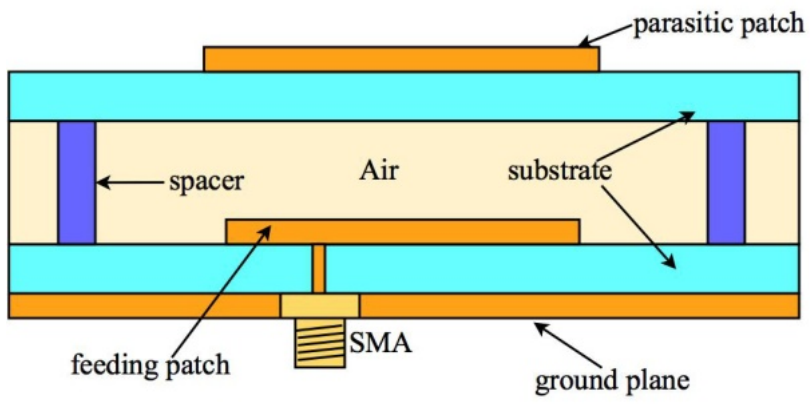

Fig. 3. Configuration parasitic stacked patch antenna [7]

\section{Gain-enhanced antenna with reflector}

A reflector is used to harvest antenna energy. Kang reported a convenient synthetic procedure. He has improved a reflector by using the particle swarm optimization (PSO) 
algorithm [5]. The reflector and ring slot antenna works to enhance the gain. The researchers set the radiation patch above the reflector. This technique was developed to reflect the back lobe field in the forward direction. It will enhance the main lobe radiation pattern and duplicate the antenna gain. The convergence becomes faster by using (PSO) algorithm, at high frequency. When the gain, axial ratio, and bandwidth are enhanced, the efficiency improves for the proposed antenna. The RF received power is considered as RF energy harvesting. As known, the Friis equation explains the relation among received and transmitted power, and the gain shown in (1).

$$
P_{r}=P_{t} G_{t}\left(\frac{\lambda}{4 \pi R}\right)^{2} G_{r}
$$

Where Pr presents received power, and Pt transmuted power. Gt and $\mathrm{Gr}$ present transmuted and received gain respectively. Moreover, $\mathrm{R}$ is the distance between the two antennas. Therefore, it can obtain a high power at the high-gain antenna. The reflected field interferes with the main radiation field. This research gives an excellent method to enhance the antenna gain. The half wavelength for the resonant band is found by calculating the total ring length. This way used to minimize the antenna size as shown in Fig. 4. That gives the optimum length and the highest current distribution in the ring. Moreover, the circular polarization improves the antenna performance. After that, the researcher cuts a slot from each corner of the radiation patch with specific positions. The benefit of the circular polarization patch gives free choices to transmit in all directions. Here, the authors work to duplicate the proposed antenna gain by setting a reflector below the patch. That will reflect the backward field and add it to the forward field. The reflector should design on 1/4 wavelength to avoid the interference and make it an improvement.

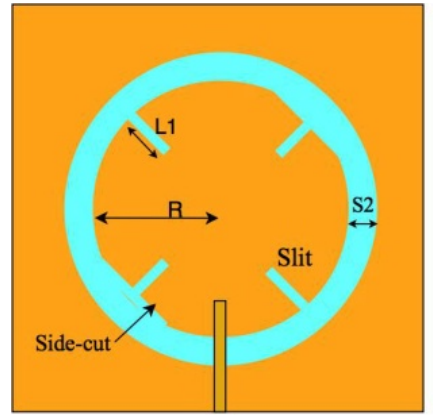

(a)

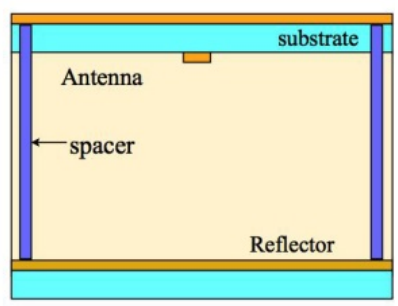

(b)
Fig. 4. The ring-slot patch antenna with reflector: (a) top; (b) side [5]

\section{Solar and RF energy harvesting of patch antenna}

Tawkt has developed a solar cell methodology on inverted F-antenna for energy harvesting [10]. The first objective is to develop two sources of stored power. Moreover, it saves a DC power, which is harvested from RF received signal. In this study, the authors illustrated two operational tasks of the proposed antenna. These transmit the data, collect the RF power, and develop a rectifier circuit with a best matching network. Fig. 5 illustrates inverted F-antenna. It has thickness $0.5 \mathrm{~mm}$ made from copper. The antenna sited between the plate of Aluminum and the solar cell on the substrate.

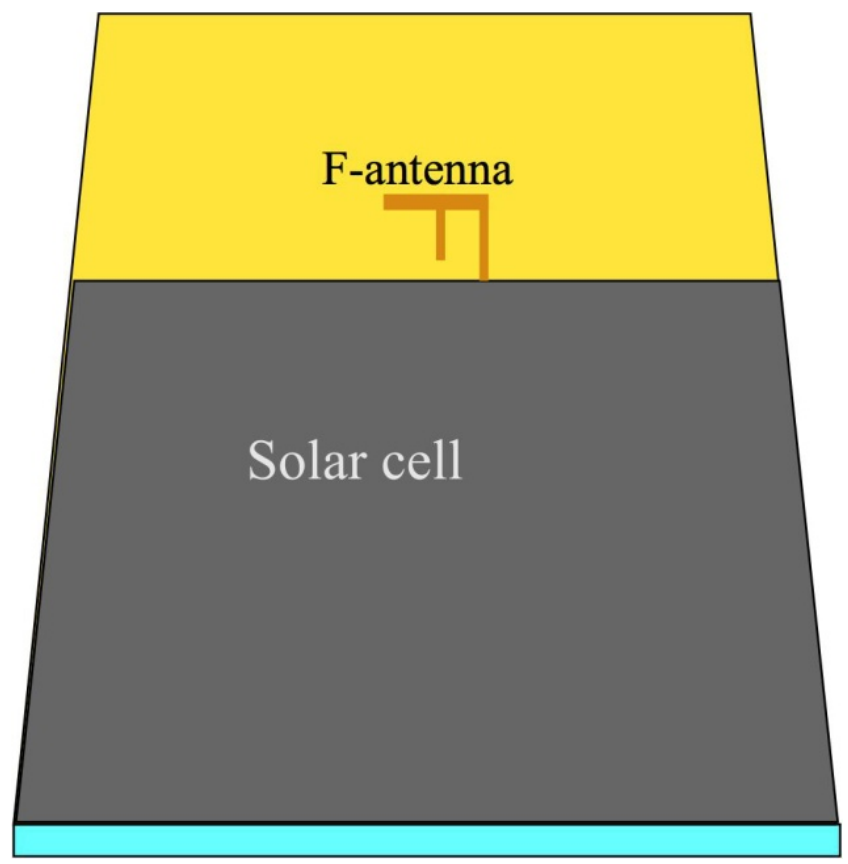

Fig. 5. The F-antenna with solar cell [10]

The main features to choose F-antenna are low profile and radiation performance. In addition, the structure of F-antenna not obscured a length of the solar cell all the time. An optimization technique is used to determine F-antenna position. However, the researcher tried to develop meshed patch antenna to avoid the changing the radiation pattern shape because the solar said effect [11]. Lastly, two scenarios are developed on both sides of the planar antenna. One of them stands at the bottom side. However, the other locates on the top aspect of the planar antenna.

\section{E. Coupled E-patch for bandwidth improvement}

In 2015, Raietal developed E-patch antenna to enhance bandwidth and efficiency [12]. The proposed antenna is presented to improve the bandwidth and the electromagnetic field by adding a couple of E-patches. They have the same dimensions and the feeding point. The bandwidth is increased by $33 \%$ when an antenna has more than one resonant frequency. E-patch antenna achieved the WLAN band. Coupled E-patches are improved to meet WLAN and ISM band. When the single E-patch antenna designed, the gain decreased sharply. Moreover, the resonance frequency reduced from $2.45 \mathrm{GHz}$ to $0.915 \mathrm{GHz}$. Practically, the highest gain of an antenna is achieved at the center resonant frequency. However, the gain was reduced slightly at the lower resonant frequency. The antenna size increased after the researchers added a couple of patches as an array antenna. After making a comparison between the single patch and array patch performance at the same frequency, the input power and gain improved. Large coupling of array E-patch used for energy harvesting. Moreover, the radiation patron becomes Omnidirectional. Fig. 6 illustrated twins of patches; they were posted beside the initially fed patch antenna. Furthermore, the E-patch will improve the antenna bandwidth. After the couple patches dimension increase, the output power improves. The incident electromagnetic radiation field is increased because of the 
radiated surface of the antenna increase. Finally, the simulation results show the enhancement of coupled patch antenna gains better than the single $\mathrm{E}$ patch antenna at the same frequency.

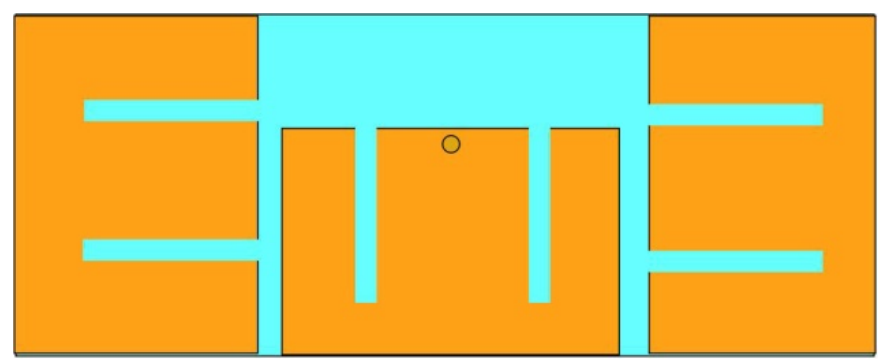

Fig. 6. Coupled E-patches with similar dimensions to the E-patch feed point[12]

\section{F. Dual-port pixel antenna}

Shen highlights a dual-port pixel antenna for energy harvesting Fig. 7 [13]. The connection between the received power and the connections of the pixels is found by using Zparameters of the antenna. Moreover, the maximum power is collected by using the genetic algorithm at the same frequency. This algorithm is applied to optimize the connection configuration of the antenna pixels. The proposed dual-port antenna gets higher power performance than a single dipole antenna with the same antenna size.

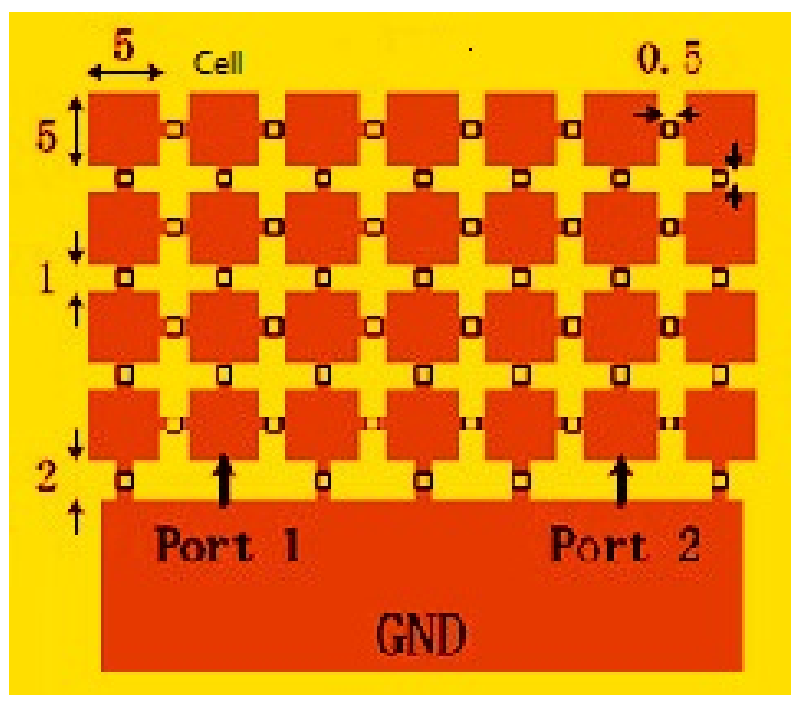

Fig. 7. The geometry of the planar dual-port pixel antenna [13]

Fig. 7 illustrated the planar dual-port pixel antenna. The authors divided the radiation surface to several pixels by $7 \times 4$ square cells. The cells are optimized due to the dimension of the ground plane. Each cell on the antenna grid has a connection to the others or with the ground plane. The black marks between the cells are $\mathrm{Q}=50$ connectors as shown in Fig. 7. For each connector, it can take two states with or without the connection. There are $2 \mathrm{Q}$ configuration states. The vector $\mathrm{x}=$ $[\mathrm{x} 1, \mathrm{x} 2, \ldots, \mathrm{xQ}] \mathrm{T}$ presented all the connection states with Xq $(\mathrm{q}=1,2, \ldots, \mathrm{Q})$, where 0 presents no connection stat and 1 presents connection stat. The two black arrows in Fig. 7 present the dual-port pixel antenna. This type of antenna is printed on the top side of the FR4 epoxy substrate that has thickness $1.6 \mathrm{~mm}$.

\section{G. Substrate Integrated Waveguide (SIW)s}

Hailin illustrates a novel dual polarization complementary split-ring resonator (CSRR) with the substrate-integratedwaveguide (SIW). These applied to fractal dual-band patch antenna for wireless energy harvesting [14]. The SIW cavity and the Giuseppe Peano fractal patch antenna were proposed. The main benefits of Giuseppe Peano fractal patches are bandwidth enhancement and minimize the antenna size. Moreover, the design achieved better directivity and higher polarization unit.

- Influence of the coupling aperture.

A couple of slot patch antennas obtained an enhancement on return losses. It integrates on the simple array antenna. In contrast, regular microstrip-fed patch and probe fed patch antennas are difficult to fabricate. An active circuit came to enhanced bandwidth and simple design. The bandwidth was enhanced by applying $\mathrm{H}$-shaped notch on a couple of patches. Moreover, the measurement shows the proposed design has efficient performance. In addition, the total radiation surface is reduced. The optimizing steps the antenna followed by setting the dual CSRR slot for the Giuseppe Peano fractal antenna.

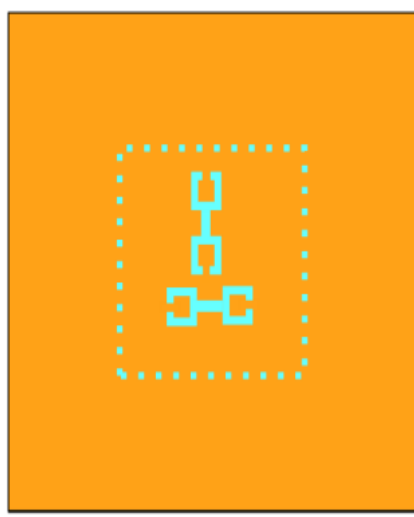

(a)

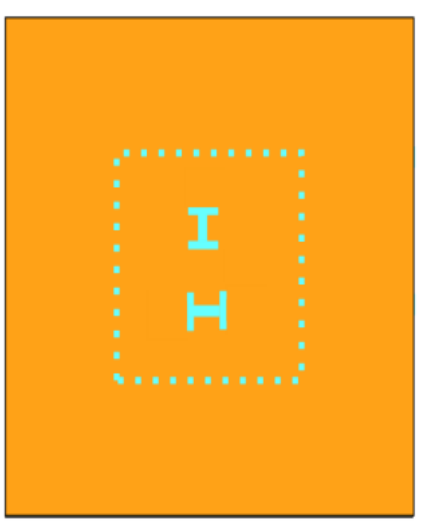

(b)
Fig. 8. The CSRR different structure [14]

In Fig. 8, the CSRR dimensions have the same length. The new structure provides a good way to reduce the antenna dimensions by using a couple of slots.

- Influence of the radiation patches shape.

The radiated wavelength depends on the effective electrical length of the patch, and the fractal geometry techniques are used. For this purpose, the total antenna size reduced to improve the fractal geometry technique. Additionally, the achieved results were better than the large antenna dimensions. Moreover, the design has been simulated by using HFSS software. After that, the simulation results compared with analytical and calculation fundamental. The reference rectangular patch and the fractal patch antenna were tested after loading CSRR slot. Fig. 9 illustrates simulation and measurement results of the proposed fractal antenna. 


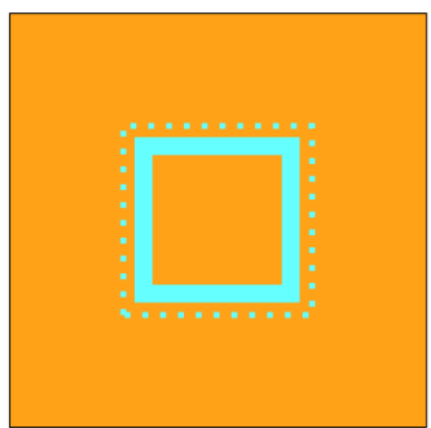

(a)

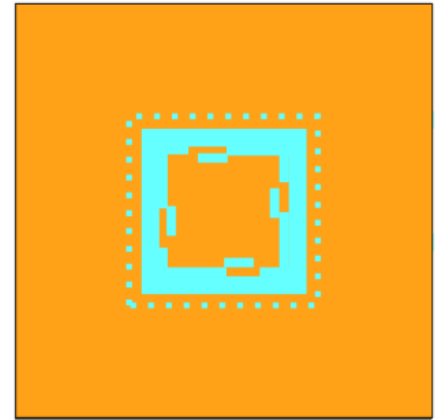

(b)
Fig. 9. SIW on fractal patch antenna [14]

A new compact CSRR-fed SIW cavity backed update the fractal patch antenna. The aim of this design is to work simultaneously on wireless energy harvesting. It is shown that the combination of fractal geometries and the CSRR feed are affected by the design of compact SIW antennas.

\section{H. Convert RF to DC power by rectenna}

Kadupitiya designed a multi-stage rectifier to increase output power with minimum antenna size [15]. The aim of this work is charged RF power electronic by harvesting the energy, which propagates in free space as a microwave signal. A rectifier circuit is connected to a patch antenna. It converted RF to DC power and used it as a second power source for the device. This circuit has an impedance match equal to the antenna impedance at the same resonant frequency. Though, the harvesting power is little; It is enough to increase the antenna efficiency. The harvesting power increased the sensor lifetime. The antenna, matching network and rectifier circuit are called a rectenna.

\section{Rectifier circuit}

The researchers choose the Villard voltage multiplier circuit because it makes double Vout from the input signal. A Schottky diode used to multiply the voltage because of it has zero voltage bias. The attractive characteristic of Schottky diode is little substrate losses and high-speed switching. The harvesting circuit used this diode by applying a single diode configuration.

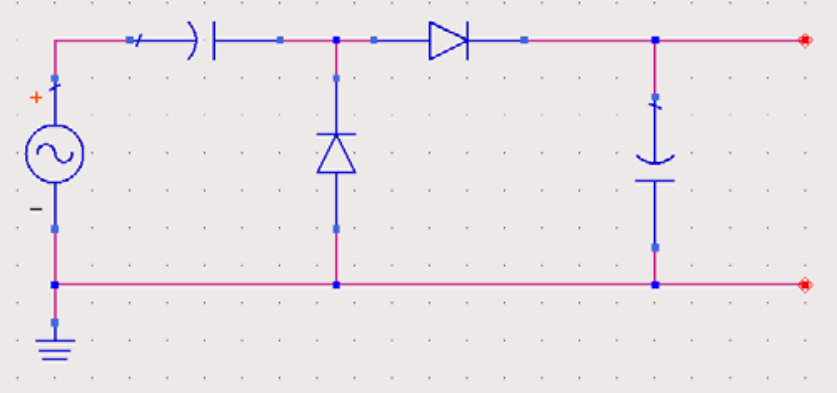

Fig. 10. Single stage rectifier circuit [15]

One stage voltage multiplier is illustrated in Fig. 10. The circuit consists of two parts capacitor and diode for rectification. The positive half cycle from the $\mathrm{RF}$ received signal can be rectified before the negative half received cycle. However, the input capacitor saved the voltage through the first-half cycle. It is transferred to the output capacitor throughout the second half cycle of the received signal. The output voltage is higher than two times of the highest RF energy.

The output of DC voltage is not accurately pure. It is an AC signal with a DC offset voltage. It is similar to DC signal superimposed by ripple satisfied. Due to this particular fact, following stages in the circuit can obtain a higher voltage than the single stage. The noise that received in the first stage will duplicate to the second stage. It will add to the total circuit noise. Therefore, any additional step will increase the output voltages depend on the received signal. Each independent circuit stage represented a single battery. In addition, it has an open-circuit load resistance $R_{L}$, output voltage $V_{0}$, internal resistance $R_{0}$, and the output voltage Vout. They are articulated by using (2).

$$
V_{\text {out }}=\frac{R_{l}}{R_{l}+R_{0}} V_{0}
$$

Generally, the negative half cycle of the received signal could decrease the output voltage. The enhancements on output voltage will double the input voltage up to the number of stages.

\section{ANALYSIS AND DISCUSSION}

Different techniques have been used to illustrate the energy harvesting on an antenna. These techniques have developed many applications. This paper shows different achievement results. The beam forming was guided on the transmitting $2 \times 2$ array antenna. The highest efficiency will achieve when the transmitter has a zero phase shift at a distance $15 \mathrm{~cm}$. In contrast, the array sets antenna 2 and 4 with $-90^{\circ}$ phase shift to control the main lobe direction by $15^{\circ}$ and achieved efficiency $3.72 \%$. The bandwidth improvement is related to the total slot length, and location. Moreover, the distribution current on the slot edge of the improvement band depends on the proposed impedance and overall slot length. After that, the gain and bandwidth were improved significantly from 5.4 MHz to 20.6 $\mathrm{MHz}$ by using the stacked slot antenna for energy harvesting. Additionally, the gain enhanced by adding a reflector bellows the single slot antenna. This reflector is used to reflect back the back-lobe field. The reflector geometry and the distance between the patch and the reflector are considered critical parameters to enhance the gain. Furthermore, a reconfigurable reflector may duplicate the gain at a particular band. Conversely, the maximum gain was duplicated from $10 \mathrm{dBi}$ to $20 \mathrm{dBi}$ by adding a coupled E-patch to the antenna. However, these couple E-patches increased the antenna size three times. On the one hand, a multi-stage rectifier circuit connected to a patch antenna. This circuit achieved ( $5 \mathrm{v}$ ) DC by harvesting the RF received signal. Nevertheless, the output power equals the input power. Indeed, the rectifier operating power is recognized as loss power. In fact, the impedance matching of a system changed when we set any additional component. So that, the RF equivalent circuit impedance should be equal to the antenna impedance at the operating band. On the other hand, the hybrid technique used to transfer RF and light to DC power. As a result, multi-course harvested the energy by connecting a 
rectifier circuit and solar cell to F-antenna. The solar cell material dielectric has to consider during the simulation. This technique has effective results at the daylight only. CSRR fractal patch with SIW improved dual-band impedance matching. SIW technique is hard to fabricate, but it reduces the antenna size. It collects the energy by using dual polarization operation bands.

\section{CONCLUSION}

This paper reviews the achievement energy harvesting on communication technologies. Our highlights were the array antenna for energy harvesting, slot patch antenna, gain enhanced, solar cells, coupled E-shaped patch antennas, dualport pixel antenna, SIW cavity-reflector patch antenna, and rectifier RF to DC energy harvesting. Different kinds of optimization schemes compared. Some overview is specified to achieve an energy harvesting. Sincerely, this paper talks about the energy harvesting techniques of communications applications, which can improve. Future work, the phase shift $90^{\circ}$ between the array antenna patches satisfied only $15^{\circ}$ controlling on the directivity of the main lobe radiation. Therefore, combining the circular polarization technique with the phase shift array antenna may improve the whole system efficiency.

\section{REFERENCES}

[1] S.-Y. Jing, S. Ali, K. She, and Y. Zhong, "State-of-the-art research study for green cloud computing," The Journal of Supercomputing, vol. 65, pp. 445-468, 2013.

[2] F. K. Shaikh and S. Zeadally, "Energy harvesting in wireless sensor networks: A comprehensive review," Renewable and Sustainable Energy Reviews, vol. 55, pp. 1041-1054, 2016.

[3] P. Nintanavongsa, "A survey on RF energy harvesting: circuits and protocols," Energy Procedia, vol. 56, pp. 414-422, 2014.

[4] Y. A. Jawhar, R. A. Abdulhasan, S. A. Hamzah and K. N. Ramli, "A New Hybrid Sub-Block Partition Scheme of PTS Technique for Reduction PAPR Performance in OFDM System," ARPN Journal of Engineering and Applied Sciences, vol. 11, pp. 4322-4332, 2016.
[5] S.-I. Kang, K.-T. Kim, S.-J. Lee, J.-P. Kim, K. Choi, and H.-S. Kim, "A Study on a Gain-Enhanced Antenna for Energy Harvesting using Adaptive Particle Swarm Optimization," Journal of Electrical Engineering and Technology, vol. 10, pp. 1780-1785, 2015.

[6] S.-F. Yang, T.-H. Huang, C.-C. Chen, C.-Y. Lu, and P.-J. Chung, "Beamforming power emitter design with $2 \times 2$ antenna array and phase control for microwave/RF-based energy harvesting," 2015 IEEE Wireless Power Transfer Conference (WPTC), pp. 1-4. 2015.

[7] T. Moura, L. Brás, P. Pinho, N. Carvalho, and R. Gonçalves, "Parasitic stacked slot patch antenna for DTT energy harvesting," 2015 IEEE International Symposium on Antennas and Propagation \& USNC/URSI National Radio Science Meeting, pp. 2445-2446. 2015.

[8] R. A. Abdulhasan, R. Alias, A. Awaleh, and A. Mumin, "Design of circular patch microstrip ultra wideband antenna with two notch filters," 2015 IEEE International Conference on Computer, Communications, and Control Technology (I4CT), pp. 464-467. 2015.

[9] R. A. Abdulhasan, M. L. Attiah, R. Alias, A. Awaleh, and A. Mumin, "Multi-State UWB Circular Patch Antenna Based on WiMAX and WLAN Notch Filters Operation," ARPN Journal of Engineering and Applied Sciences, vol. 10, p. 5, 2015.

[10] Y. Tawk, J. Costantine, and C. Christodoulou, "An inverted-F antenna integrated with solar cells for energy harvesting," 2015 9th European Conference on Antennas and Propagation (EuCAP), pp. 1-2. 2015.

[11] T. W. Turpin and R. Baktur, "Meshed patch antennas integrated on solar cells," IEEE Antennas and Wireless Propagation Letters, vol. 8, pp. 693696, 2009.

[12] G. Rai, A. Johari, and R. Shamim, "A wideband coupled E-shaped patch antenna for RF energy harvesting," 2015 International Conference on Signal Processing and Communication (ICSC), pp. 390-394. 2015.

[13] S. Shen and R. D. Murch, "Designing dual-port pixel antenna for ambient RF energy harvesting using genetic algorithm," 2015 IEEE International Symposium on Antennas and Propagation \& USNC/URSI National Radio Science Meeting, pp. 1286-1287. 2015.

[14] H. Cao, F. Jiang, J. Liu, W. Cai, M. Tang, X. Tan, et al., "A CSRR-fed SIW cavity-backed fractal patch antenna for wireless energy harvesting and communication," Sensors, vol. 15, pp. 21196-21203, 2015.

[15] J. Kadupitiya, T. Abeythunga, P. Ranathunga, and D. De Silva, "Optimizing RF energy harvester design for low power applications by integrating multi stage voltage doubler on patch antenna," 2015 8th International Conference on Ubi-Media Computing (UMEDIA), pp. 335-338, 2015j 